\title{
CRITÉRIOS PARA CLASSIFICAÇÃO VISUAL DE PEÇAS ESTRUTURAIS DE Pinus $S p$
}

\section{MARCELO RODRIGO CARREIRA}

Dissertação apresentada à Escola de Engenharia de São Carlos, da Universidade de São Paulo, como parte dos requisitos necessários à obtenção do título de Mestre em Engenharia de Estruturas.

ORIENTADOR: Prof. Dr. Antônio Alves Dias 
Aos meus pais Alcides e Lourdes, e a minha namorada Elizângela... 


\section{AGRADECIMENTOS}

A Deus, pela saúde e pela oportunidade de estudar .

Ao Professor Antônio Alves Dias, pela amizade e excelente orientação fornecida durante a elaboração deste trabalho.

Ao Professor Carlito Calil Jr., pela sugestão do tema, pela co-orientação fornecida e pela amizade.

Ao amigo Andrés Batista Cheung, pela colaboração nos trabalhos e pela amizade.

A Mariano Martinez Espinosa, pelas dicas com as análises estatísticas do trabalho.

A todos os amigos do LaMEM, que de alguma forma colaboraram direta ou indiretamente para a produção deste trabalho.

A meus pais Alcides e Lourdes, pelo apoio moral e financeiro fornecido durante todo o curso de mestrado.

Ao meu amor Elizângela, pela paciência e pelo apoio nos momentos difíceis.

Aos professores Cesar Ballarotti e Everaldo Pletz pelo incentivo.

A Battistella Indústria e Comércio de Madeiras pelo fornecimento de toda a madeira utilizada neste trabalho.

Ao IBAMA por permitir o uso da máquina MSR.

Ao CNPq pela bolsa de estudos concedida. 


\section{SUMÁRIO}

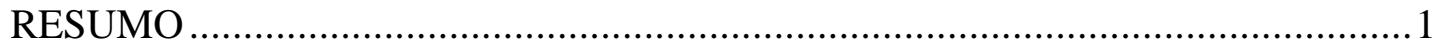

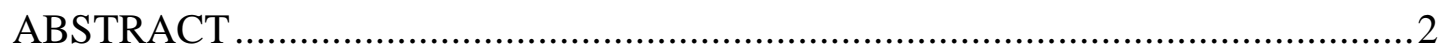

1 INTRODUÇÃ

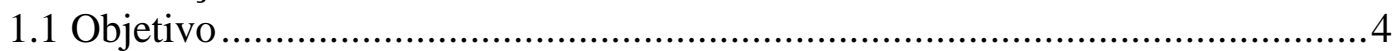

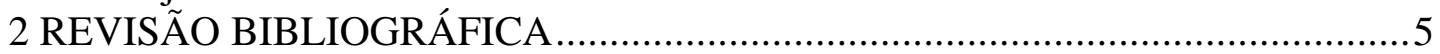

2.1 Classificação de peças estruturais de madeira no Brasil - estado da arte ..........5

2.2 Métodos de classificação estrutural da madeira ......................................... 10

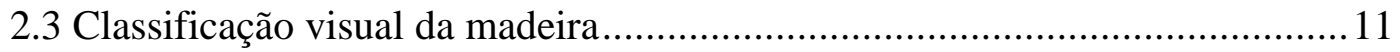

2.3.1 Histórico da classificação visual ....................................................... 11

2.3.2 Componentes de uma classificação visual..............................................13

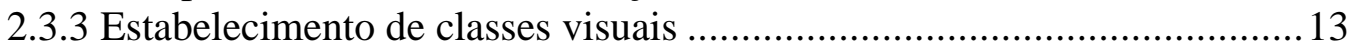

2.3.4 Determinação das propriedades de resistência .......................................34

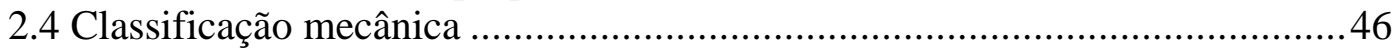

2.4.1 Histórico da classificação mecânica................................................... 47

2.4.2 Componentes de uma classificação mecânica .......................................49

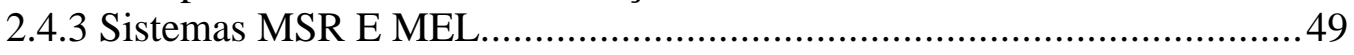

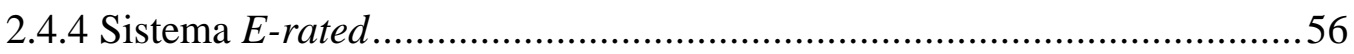

2.4.5 Avaliação não destrutiva pela técnica de vibração transversal .................60

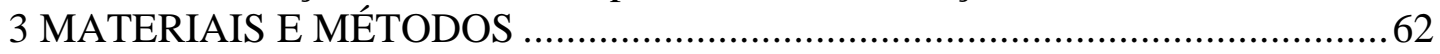

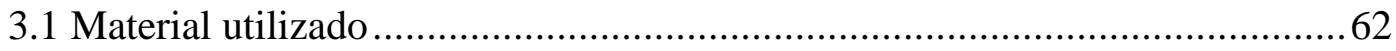

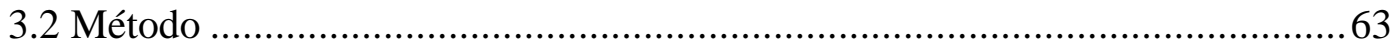

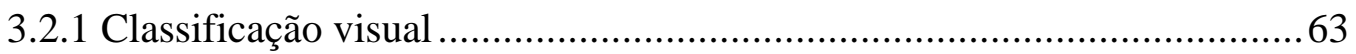

3.2.2 Classificação mecânica na máquina MSR .............................................64

3.2.3 Classificação mecânica por vibração transversal...................................66

3.2.4 Teste de flexão estática em relação ao eixo de menor inércia .................67

3.2.5 Teste de flexão estática em relação ao eixo de maior inércia...................68

3.2.6 Teste de compressão paralela às fibras em corpos-de-prova.................... 71

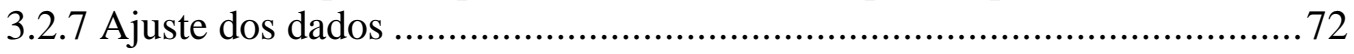

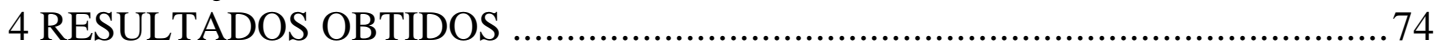

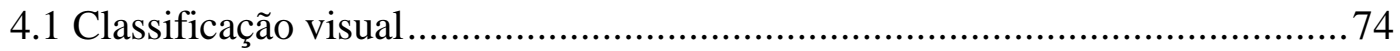

4.2 Valores de MOE em relação ao eixo de menor inércia..................................74

4.3 MOR e MOE segundo o eixo de maior inércia ............................................81

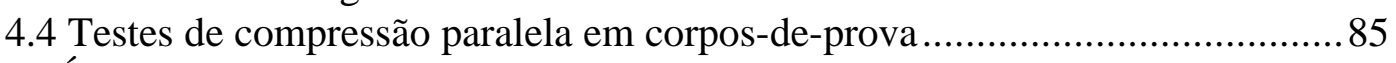

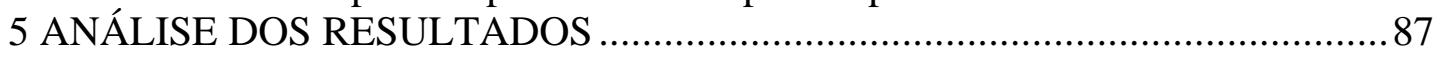

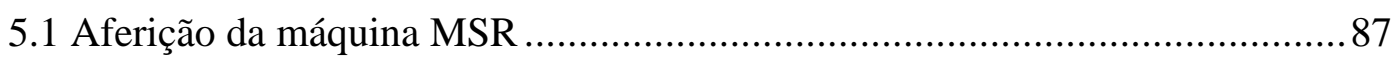

5.2 Aferição do equipamento de vibração transversal ...................................... 95

5.3 Verificação da influência da disposição das pranchas na determinação do MOE

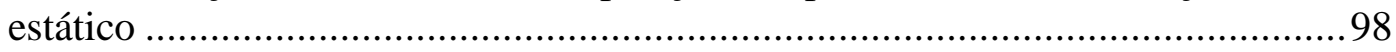

5.4 Análise dos resultados do MOE em relação ao eixo de maior inércia............ 106

5.5 Análise dos resultados do MOR em relação ao eixo de maior inércia .......... 111

5.6 Análise da correlação entre MOR e MOE em relação ao eixo de maior inércia.

5.7 Análise da correlação entre o MOR e o MOE em relação ao eixo de menor inércia.

5.8 Determinação do Valor Característico do MOR e do valor médio do MOE em relação ao eixo de maior inércia para cada classe. 
5.9 Análise da correlação entre $\mathrm{E}_{\mathrm{co}}$ e $\mathrm{f}_{\mathrm{co}}$ para os corpos-de-prova. 125

5.10 Análise da correlação entre $\mathrm{f}_{\mathrm{co}}$ e $\rho_{\text {ap }}$ para os corpos-de-prova 127

5.11 Análise da correlação entre $\mathrm{E}_{\mathrm{co}}$ e $\rho_{\mathrm{ap}}$ para os corpos-de-prova. 129

5.12 Determinação das propriedades de resistência e rigidez à compressão paralela em corpos-de-prova .... 131

5.13 Comparação das resistências à compressão paralela entre os corpos-de-prova.

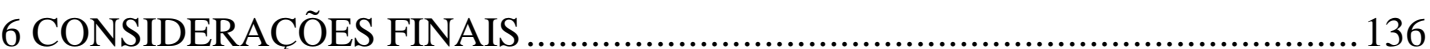

7 ANEXO A - CRITÉRIOS PARA A LIMITAÇÃO DOS DEFEITOS ................. 142

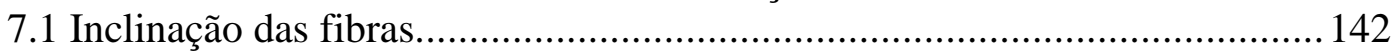

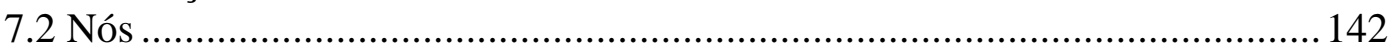

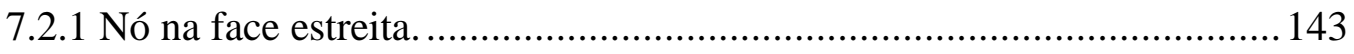

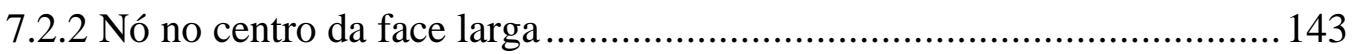

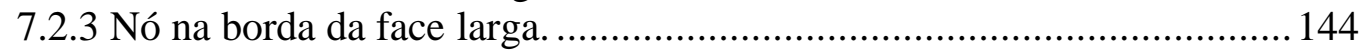

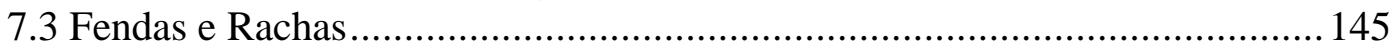

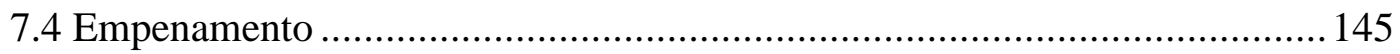

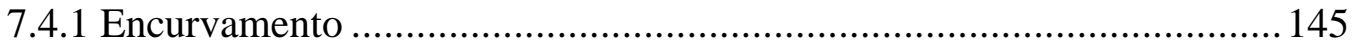

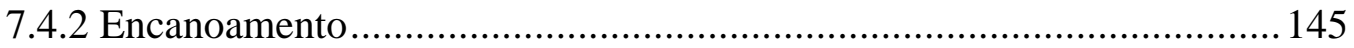

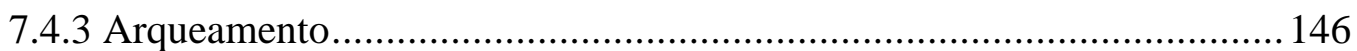

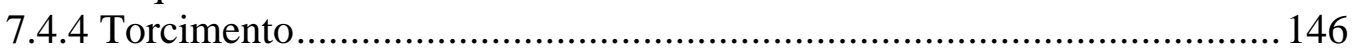

8 ANEXO B - Códigos de ruptura utilizados nos testes de flexão estática e

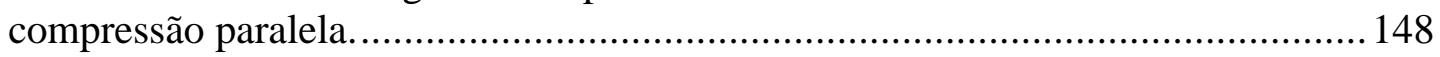

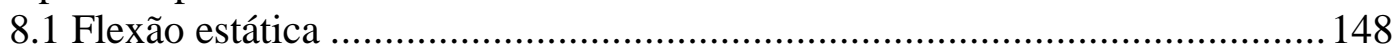

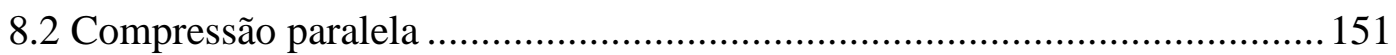

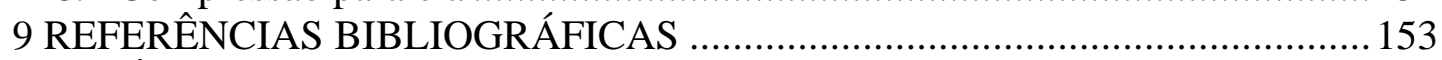

10 APÊNCICE A - EXEMPLO DE REGRAS DE CLASSIFICAÇÃO VISUAL ESTRUTURAL PARA MADEIRA DE CONÍFERAS …................................... 158 11 APENDICE $\mathrm{B}$ - INFLUÊNCIA DAS CARACTERÍSTICAS DE CRESCIMENTO NA RESISTÊNCIA........................................................ 160

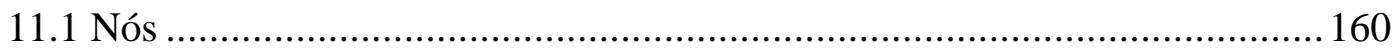

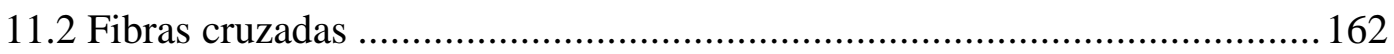

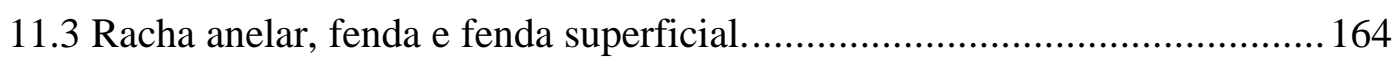

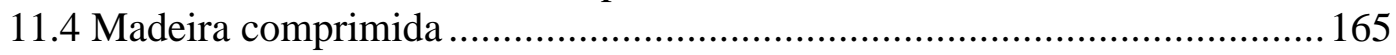

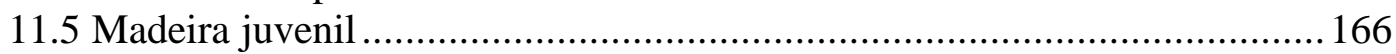

12 APÊNDICE C - CONSIDERAÇÕES ADICIONAIS PARA O EMPREGO DOS SISTEMAS DE CLASSIFICAÇÃO VISUAL E MECÂNICA NA INDÚSTRIA DE

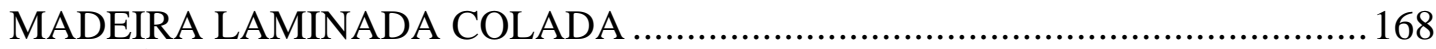

12.1 ÍNDICES DE RESISTÊNCIA (Stress index) .......................................... 169

12.1.1 Madeira visualmente classificada ...................................................... 169

12.1.2 Madeira classificada pelo sistema E-rated ....................................... 171

12.2 FATORES MODIFICADORES DA RESISTÊNCIA............................. 171

12.2.1 Madeira visualmente classificada................................................... 172

12.2.2 Madeira classificada pelo sistema E-rated ..................................... 175

13 APÊNDICE D - Valores das propriedades de resistência e rigidez para o Southern

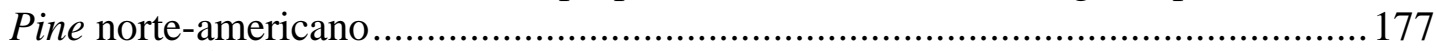

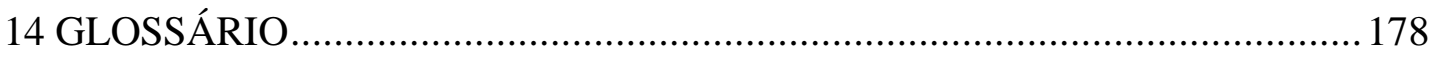




\section{LISTA DE FIGURAS}

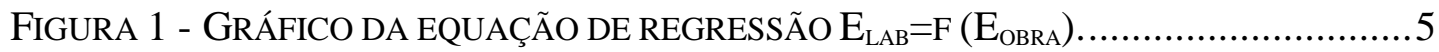

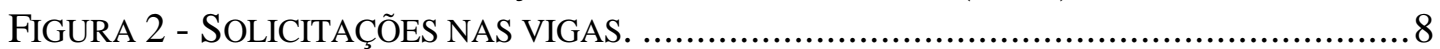

FIGURA 3 - MEDIÇÃO DA INCLINAÇÃO DAS FIBRAS DE UMA PEÇA DE MADEIRA. .........20

FIGURA 4 - IDENTIFICAÇÃO DOS NÓS EM UMA PEÇA DE MADEIRA. ............................20

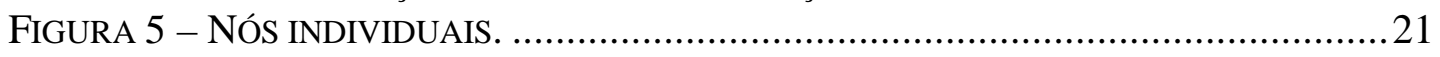

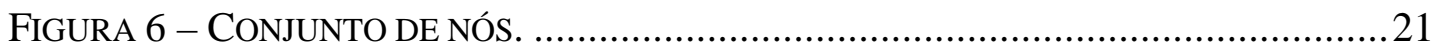

FIGURA 7 - NÓ NA BORDA DE UMA PEÇA..................................................... 21

FIGURA 8 - MEDIÇÃO DE UM NÓ NA FACE ESTREITA. ............................................ 22

FIGURA 9 - MEDIÇÃO DE UM NÓ QUE APARECE NAS DUAS FACES DE UMA PEÇA SEM

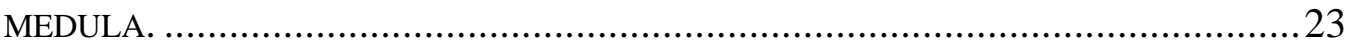

FIGURA 10 - MEDIÇÃO DE UM NÓ NA FACE LARGA...............................................23

FIGURA 11 - MEDIÇÃO DE UM NÓ DE CANTO EM PEÇA SEM MEDULA. ..........................23

FIGURA 12 - MEDIÇÃO DE UM NÓ DE CANTO EM PEÇA CONTENDO MEDULA.................24

FIGURA 13 - MEDIÇÃO DO DIÂMETRO DOS NÓS EM UMA VIGA. ..................................25

FIGURA 14 - MEDIÇÃO DO DIÂMETRO DOS NÓS EM UMA COLUNA. ..............................26

FIGURA 15 - MEDIÇÃO DO DIÂMETRO DOS NÓS EM TÁBUAS.....................................27

FIGURA 16 - FORMA DE MEDIR UMA RACHA CONFORME ASTM D245-93 ................28

FIGURA 17 - FORMAS DE MEDIR UMA FENDA CONFORME ASTM D245-93 ...............29

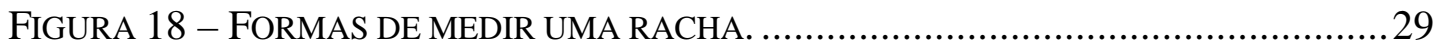

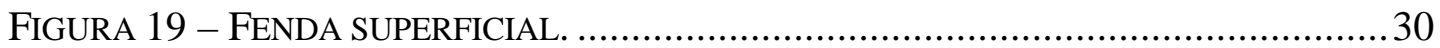

FIGURA 20 - FENDA QUE ATRAVESSA A PEÇA EM ESPESSURA.................................. 30

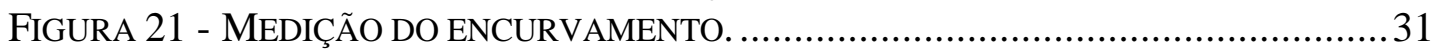

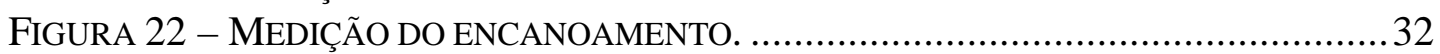

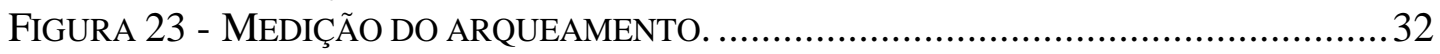

FIGURA 24 - MEDIÇÃO DO TORCIMENTO........................................................... 32

FIGURA 25- LINHA RADIAL REPRESENTATIVA EM PEÇAS CONTENDO MEDULA............. 33

FIGURA 26 - LINHA RADIAL REPRESENTATIVA EM PEÇAS SEM MEDULA........................ 34

FIGURA 27 - HISTOGRAMA DE FREQÜÊNCIAS RELATIVAS. ................................... 40

FIGURA 28 - HISTOGRAMA DE FREQÜÊNCIAS RELATIVAS PARA OS DADOS

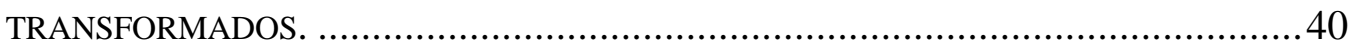

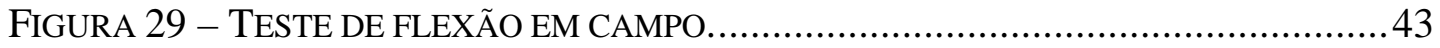

FIGURA 30 - MÁQUINA DE FLEXÃO UTILIZADA NO PROGRAMA IN-GRADE ALOJADA DENTRO DO PORTA-MALAS DE UM VEÍCULO DE PASSEIO...................................4 44

FIGURA 31 - ESQUEMA DO FUNCIONAMENTO DE UMA MÁQUINA MSR .......................48

FIGURA 32 - ESTIMATIVA DA RESISTÊNCIA POR ANÁLISE DE REGRESSÃO. ...................54

FIGURA 33 - RELAÇÃO TÍPICA ENTRE O MÓDULO DE ELASTICIDADE E O MÓDULO DE

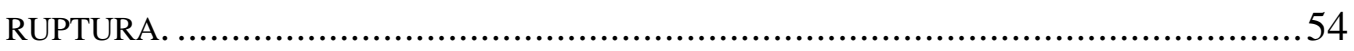

FIGURA 34 - RELAÇÃO ENTRE O MOR E O MOE ESTÁTICO EM RELAÇÃO AO EIXO DE

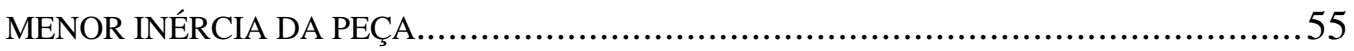

FIGURA 35 - ESTIMATIVA DO MOE MÍNIMO PARA O ENQUADRAMENTO NAS CLASSES

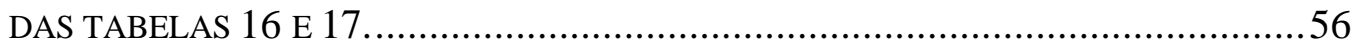

FIGURA 36 - SISTEMA MASSA-MOLA E VIGA VIBRANDO TRANSVERSALMENTE............60

FIGURA 37 - ESQUEMA DE UM EQUIPAMENTO PARA A DETERMINAÇÃO DO MOE POR

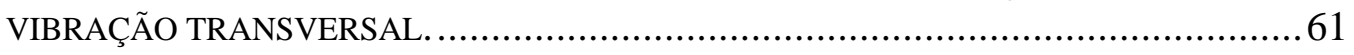

FIGURA 38 - CLASSIFICAÇÃO VISUAL E DETALHE DO GABARITO DE ACRÍLICO............64

FIGURA 39 - ELEMENTOS DA MÁQUINA DE CLASSIFICAÇÕES...................................65 
FIGURA 40 - DETALHES DA CALIBRAÇÃO DA MÁQUINA MSR. 65

FIGURA 41 - DESENVOLVIMENTO DA CLASSIFICAÇÃO COM A MÁQUINA MSR ...........66

FIGURA 42 - EQUIPAMENTO DE VIBRAÇÃO TRANSVERSAL UTILIZADO NOS TESTES.....67

FIGURA 43 - TESTE DE FLEXÃO ESTÁTICA EM RELAÇÃO AO EIXO DE MENOR INÉRCIA

DAS PRANCHAS E DETALHE DO APOIO..

FIGURA 44 - ARRANJO DOS TESTES DE FLEXÃO ESTÁTICA EM RELAÇÃO AO EIXO DE MAIOR INÉRCIA.

FIGURA 45 - DETALHE DOS CONTRAVENTAMENTOS. .......................................... 70

FIGURA 46 - TRANSDUTORES DE DESLOCAMENTO E CÉLULA DE CARGA....................72

FIGURA 47 - DIAGRAMA DE DISPERSÃO E RETA DE REGRESSÃO ENTRE MOE ESTÁTICO E MOE MSR.

FIGURA 48 - GRÁFICO DOS RESÍDUOS CONTRA VALORES ESTIMADOS PARA O MOE MSR.

FIGURA 49 - GRÁFICO DOS RESÍDUOS CONTRA OS ESCORES NORMAIS........................ 90

FIGURA 50 - DETERMINAÇÃO DO $\lambda_{\text {ótimo PARA O MOE }}$ MSR . ...................................... 91

FigURA 51 - DETERMINAÇÃO DO $\lambda_{\text {ótIMO }}$ PARA O MOE

FIGURA 52 - RESÍDUOS CONTRA VALORES ESTIMADOS PARA OS DADOS TRANSFORMADOS.

FIGURA 53 - RESÍDUOS CONTRA ESCORES NORMAIS PARA OS DADOS TRANSFORMADOS.

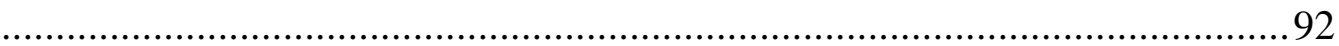

FIGURA 54 - HISTOGRAMA DOS RESÍDUOS PARA OS DADOS TRANSFORMADOS. ...........93

FIGURA 55 - DIAGRAMA DE DISPERSÃO E RETA DE REGRESSÃO ENTRE MOE ESTÁTICO

E MOE MSR, PARA OS DADOS TRANSFORMADOS........................................93

FIGURA 56 - DiAGRAMA DE DISPERSÃO E RETA DE REGRESSÃO ENTRE MOE ESTÁtico E

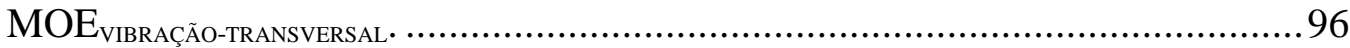

FIGURA 57 - RESÍDUOS CONTRA VALORES ESTIMADOS PARA OS DADOS DO MOE OBTIDO NA VIBRAÇÃO TRANSVERSAL. ..................................................... 96

FIGURA 58 - RESÍDUOS CONTRA ESCORES NORMAIS PARA OS DADOS DO MOE OBTIDO NA VIBRAÇÃO TRANSVERSAL.

FIGURA 59 - HISTOGRAMA DOS RESÍDUOS PARA OS DADOS DO MOE OBTIDO NA VIBRAÇÃO TRANSVERSAL.

FIGURA 60 - GRÁFICO DE RESÍDUOS CONTRA VALORES ESTIMADOS PARA OS DADOS AGRUPADOS DO MOE DA CLASSE N²-ND.

FIGURA 61 - GRÁFICO DE RESÍDUOS CONTRA OS ESCORES NORMAIS PARA OS DADOS AGRUPADOS DO MOE DA CLASSE N²-ND

FIGURA 62 - DETERMINAÇÃO DO $\lambda_{\text {ótImo }}$ PARA OS DADOS AGRUPADOS DO MOE DA CLASSE N ${ }^{\circ} 2-N D$

FIGURA 63 - GRÁFICO DE RESÍDUOS CONTRA VALORES ESTIMADOS PARA OS DADOS TRANSFORMADOS E AGRUPADOS DO MOE DA CLASSE N²-ND.

FIGURA 64 - GRÁFICO DE RESÍDUOS CONTRA OS ESCORES NORMAIS PARA OS DADOS TRANSFORMADOS DO MOE DA CLASSE N²-ND.

FIGURA 65 - TESTE DE NORMALIDADE PARA OS DADOS TRANSFORMADOS DO MOE DA CLASSE N 2 -ND.

FIGURA 66 - GRÁFICO DE RESÍDUOS CONTRA VALORES ESTIMADOS PARA OS DADOS DO MOE.

FIGURA 67 - GRÁFICO DE PROBABILIDADE NORMAL DOS RESÍDUOS. ....................... 107

FIGURA 68 - TESTE DE NORMALIDADE PARA OS DADOS DO MOE........................... 107

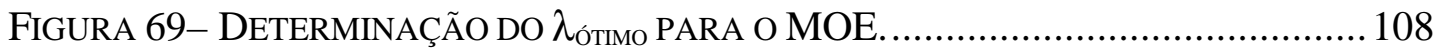


FIGURA 70 - GRÁFICO DE RESÍDUOS CONTRA VALORES ESTIMADOS PARA OS DADOS DO MOE TRANSFORMADOS PELA EQUAÇÃO 30. 108

FIGURA 71 - GRÁFICO DE PROBABILIDADE NORMAL PARA OS DADOS DA TABELA 32.

FIGURA 72 -TESTE DE NORMALIDADE DE SHAPIRO-WILK PARA O MOE TRANSFORMADO.

FIGURA 73 - RESÍDUOS CONTRA VALORES ESTIMADOS PARA OS DADOS DO MOR.... 111

FIGURA 74 - GRÁFICO DOS RESÍDUOS CONTRA OS ESCORES NORMAIS PARA OS DADOS DO MOR.

FIGURA 75 - TESTE DE NORMALIDADE PARA OS DADOS DO MOR. 112

FIGURA 76 - DETERMINAÇÃO DO $\lambda_{\text {ÓtIMO }}$ PARA O MOR 113

FIGURA 77 - RESÍDUOS CONTRA VALORES ESTIMADOS PARA OS DADOS TRANSFORMADOS DO MOR.

FIGURA 78 - GRÁFICO DOS RESÍDUOS CONTRA OS ESCORES NORMAIS PARA OS DADOS DO MOR.

FIGURA 79 - TESTE DE NORMALIDADE PARA OS DADOS TRANSFORMADOS DO MOR. 114 FIGURA 80 - DiAGRAMA DE DISPERSÃO E RETA DE REGRESSÃO ENTRE MOE E MOR.

FIGURA 81 - GRÁFICO DOS RESÍDUOS CONTRA VALORES ESTIMADOS PARA O MOR. 116 FIGURA 82 - GRÁFICO DE PROBABILIDADE NORMAL DOS RESÍDUOS. ....................... 117 FIGURA 83 - HISTOGRAMA DOS RESÍDUOS. 117

FIGURA 84 - DIAGRAMA DE DISPERSÃO E RETA DE REGRESSÃO ENTRE MOE E MOR.

FIGURA 85 - GRÁFICO DOS RESÍDUOS CONTRA VALORES ESTIMADOS PARA O MOR. 119

FIGURA 86 - GRÁFICO DOS RESÍDUOS CONTRA OS ESCORES NORMAIS...................... 120

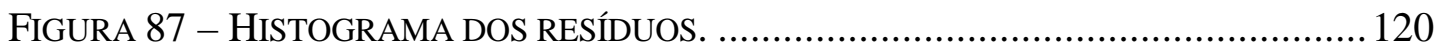

FIGURA 88 - HISTOGRAMA DE FREQÜÊNCIAS DO MOR PARA A CLASSE Nº2-ND. .... 121

FIGURA 89 - HISTOGRAMA DE FREQÜÊNCIAS DO MOR PARA A CLASSE Nº2-D........ 122

FIGURA 90 - HISTOGRAMA DE FREQÜÊNCIAS DO MOR PARA A CLASSE SS-ND....... 122

FIGURA 91 - HISTOGRAMA DE FREQÜÊNCIAS DO MOR PARA A CLASSE SS-D. .........122

FIGURA 92 - TESTE DE NORMALIDADE PARA O MOR DA CLASSE Nº2-ND................123

FIGURA 93 - TESTE DE NORMALIDADE PARA O MOR DA CLASSE Nº2-D. .................123

FIGURA 94 - TESTE DE NORMALIDADE PARA O MOR DA CLASSE SS-ND ..................1 124

FIGURA 95 - TESTE DE NORMALIDADE PARA O MOR DA CLASSE SS-D ....................124

FIGURA 96 - DIAGRAMA DE DISPERSÃO E RETA DE REGRESSÃO ENTRE E $\mathrm{CO}_{\mathrm{CO}}$ E $F_{\mathrm{CO}} \ldots . . .125$

FIGURA 97 - GRÁFICO DOS RESÍDUOS CONTRA VALORES ESTIMADOS PARA $F_{\mathrm{CO}} \ldots \ldots . . .126$

FIGURA 98 - GRÁFICO DOS RESÍDUOS CONTRA OS ESCORES NORMAIS.......................126

FIGURA 99 - DIAGRAMA DE DISPERSÃO E RETA DE REGRESSÃO ENTRE $\rho_{\text {AP }}$ E $F_{\mathrm{CO}} \ldots \ldots .127$

FIGURA 100 - GRÁFICO DOS RESÍDUOS CONTRA VALORES ESTIMADOS PARA $F_{\mathrm{CO}} \ldots . . .128$

FIGURA 101 - GRÁFICO DOS RESÍDUOS CONTRA OS ESCORES NORMAIS....................128

FIGURA 102 - DIAGRAMA DE DISPERSÃO E RETA DE REGRESSÃO ENTRE $\rho_{\text {AP }}$ E MOE CO. $_{\text {. }}$

FIGURA 103 - GRÁFICO DOS RESÍDUOS CONTRA VALORES ESTIMADOS PARA MOE

FIGURA 104 - GRÁFICO DOS RESÍDUOS CONTRA OS ESCORES NORMAIS..................... 130

FIGURA 105 - TESTE DE NORMALIDADE PARA OS DADOS TRANSFORMADOS DE $F_{\mathrm{CO}} . .132$

FIGURA 106 - TESTE DE NORMALIDADE NOS DADOS DE $F_{\mathrm{CO}}$ PARA OS CORPOS-DEPROVA. 
FIGURA 107 - GRÁFICO DOS RESÍDUOS CONTRA OS VALORES ESTIMADOS PARA OS DADOS DE $F_{\mathrm{CO}}$....

FIGURA 108 - GRÁFICO DOS RESÍDUOS CONTRA OS ESCORES NORMAIS PARA OS DADOS

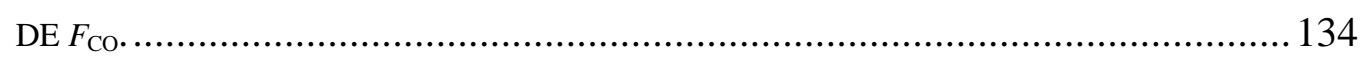

FIGURA 109 - PEÇAS DE MADEIRA COM ELEVADA DENSIDADE ............................... 138

FIGURA 110 - TIPOS DE NÓS E MEDIÇÕES PARA DESCRIÇÃO DO CÓDIGO.................... 149

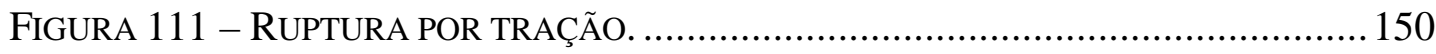

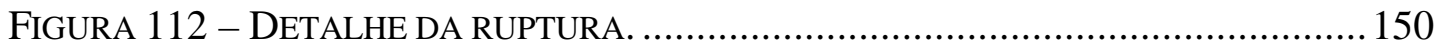

FIGURA 113 - RUPTURA POR TRAÇÃO NO NÓ.................................................. 150

FIGURA 114 - RUPTURA FRÁGIL POR TRAÇÃO. .................................................... 150

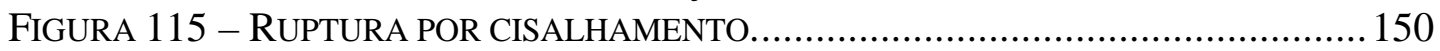

FIGURA 116 - RUPTURA DEVIDO À INCLINAÇÃO DE FIBRAS.................................... 150

FIGURA 117 - RUPTURA POR COMPRESSÃO (ESMAGAMENTO DE FIBRAS) . ................151

FIGURA 118 - DETALHE DO ESMAGAMENTO DAS FIBRAS. ….................................. 151

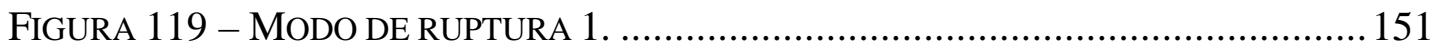

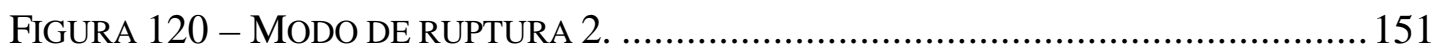

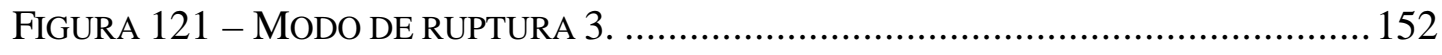

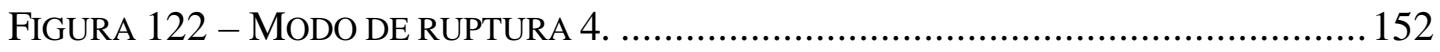

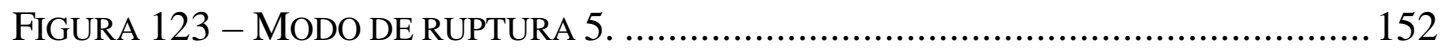

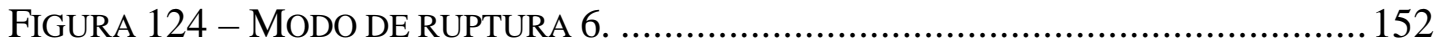

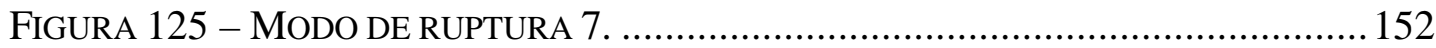

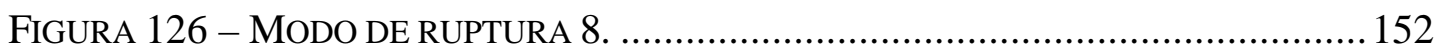

FIGURA 127 - EFEITO DO NÓ NA RESISTÊNCIA DA MADEIRA..................................... 160

FIGURA 128 - TIPOS DE NÓS LIMITADOS NA CLASSIFICAÇÃO VISUAL: (A) CENTRO DA

FACE LARGA; (B) BORDA DA FACE LARGA E (C) FACE ESTREITA. ....................... 161

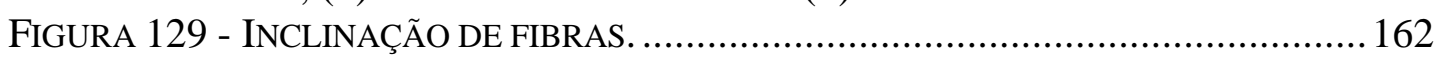

FIGURA 130 - EFEITO DAS FIBRAS INCLINADAS NA RESISTÊNCIA À FLEXÃO............... 163

FIGURA 131 - EFEITO DAS FIBRAS INCLINADAS NA RESISTÊNCIA A COMPRESSÃO...... 163

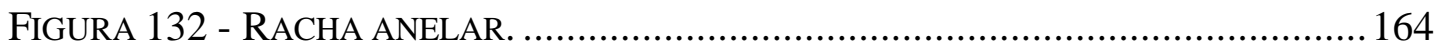

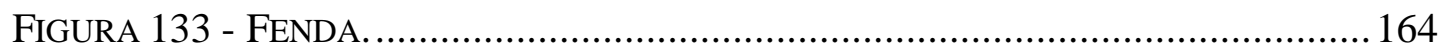

FIGURA 134 - SEÇÃO TRANSVERSAL DE UMA TORA CONTENDO MADEIRA COMPRIMIDA.

165

FIGURA 135 - PEÇA DE MADEIRA SERRADA COM MADEIRA COMPRIMIDA. ..................... 166

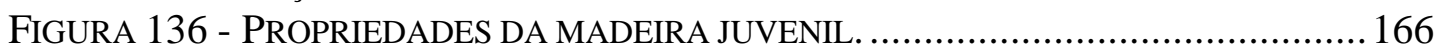

FIGURA 137 - REGIÃO DE MADEIRA JUVENIL, DE TRANSIÇÃO E ADULTA.................... 167 


\section{LISTA DE TABELAS}

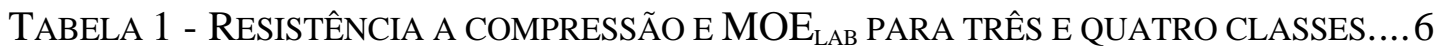
TABELA 2 - CLASSES DE RESISTÊNCIA PARA AS SEIS ESPÉCIES DE DICOTILEDÔNEAS. ....6 TABELA 3 -ESPÉCIES COMERCIAIS E REGIÃO ONDE FORAM OBTIDAS. ........................... TABELA 4 -VALORES DE $E_{\text {M,12\% }}$ (MPA) NOS PONTOS 1 E 2 DAS VIGAS DE 4,00M........... 9 TABELA 5 - VALORES DE $\mathrm{E}_{\mathrm{M}, 12 \%}$ (MPA) NOS PONTOS 1E 2 DAS VIGAS DE 5,00M........... 9

TABELA 6 - VALORES DE $\mathrm{E}_{\mathrm{M}, 12 \%}$ (MPA) NOS PONTOS 1 E 3 DAS VIGAS DE 5,00M.......... 9

TABELA 7 - VALORES DE $\mathrm{E}_{\mathrm{M}, 12 \%}$ (MPA) NOS PONTOS 2 E 3 DAS VIGAS DE 5,00M......... 10

TABELA 8 - NORMAS NORTE-AMERICANAS PARA CLASSIFICAÇÃO ESTRUTURAL DA

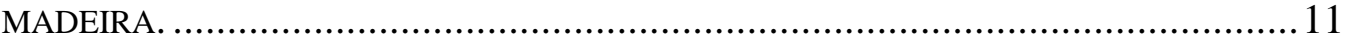

TABELA 9 - AGÊNCIAS NORTE-AMERICANAS DE CLASSIFICAÇÃO DA MADEIRA

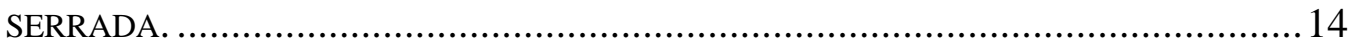

TABELA 10 - CLASSES VISUAIS DESCRITAS NA NATIONAL GRADING RULE................... 15

TABELA 11 - GRUPOS DE ESPÉCIES E AGÊNCIAS DE CLASSIFICAÇÃO NORTE-

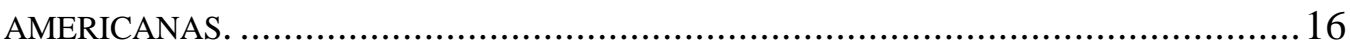

TABELA 12- CLASSES DE DENSIDADE DEFINIDAS NA ASTM D245-93 …....................33

TABELA 13 - PROPRIEDADES DE PEÇAS DE 140MM X 140MM DE SOUTHERN PINE. .......38

TABELA 14 - PROPRIEDADES EXPERIMENTAIS E USUAIS PARA COLUNAS DE SOUTHERN

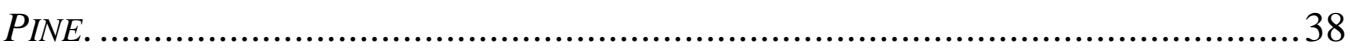

TABELA 15 - FATOR DE POSIÇÃO PARA A RESISTÊNCIA A FLEXÃO DE PEÇAS

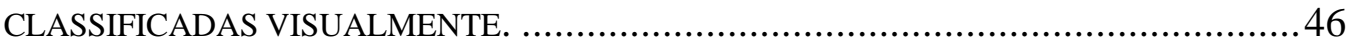

TABELA 16 - CLASSES COMUNS DA CLASSIFICAÇÃO MSR ...................................50

TABELA 17 - CLASSES COMUNS DA CLASSIFICAÇÃO MEL.......................................51

TABELA 18 - FATOR DE POSIÇÃO PARA A RESISTÊNCIA A FLEXÃO DE PEÇAS CLASSIFICADAS MECÂNICAMENTE. ............................................................51

TABELA 19 - COMPARAÇÃO DE PROPRIEDADES MECÂNICA DA CLASSIFICAÇÃO MECÂNICA COM A CLASSIFICAÇÃO VISUAL.....................................................53

TABELA 20 - EQUIVALÊNCIA APROXIMADA DAS DIMENSÕES DOS NÓS DE BORDA PARA A CLASSIFICAÇÃO MECÂNICA E VISUAL. ......................................................5

TABELA 21 - DIMENSÕES MÁXIMAS ADMISSÍVEIS DOS NÓS LOCALIZADOS NA BORDA DAS PEÇAS PARA A CLASSIFICAÇÃO MECÂNICA E VISUAL .................................58

TABELA 22 - VALOR MÍNIMO DO MOE PARA CADA CLASSE. .....................................59

TABELA 23 - LIMITAÇÕES NOS DEFEITOS EM CADA CLASSE PARA PEÇAS DE SEÇÃO TRANSVERSAL DE $3,5 \mathrm{CM} \times 12,5 \mathrm{CM}$. E COMPRIMENTO DE 2,60M.......................64

TABELA 24-CÓDIGO DE CORES E OS CORRESPONDENTES INTERVALOS DE MOE.........66

TABELA 25 - PEÇAS RECOLHIDAS PARA ANÁLISE. ....................................................74

TABELA 26 - DADOS OBTIDOS NA CLASSIFICAÇÃO VISUAL E MECÂNICA DO LOTE DE

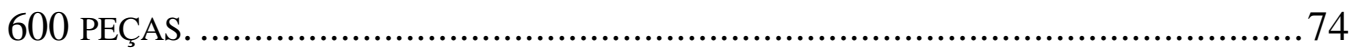

TABELA 27 - ESTATÍSTICA DESCRITIVA DOS RESULTADOS DA CLASSIFICAÇÃO MECÂNICA COM A MÁQUINA MSR E COMO EQUIPAMENTO DE VIBRAÇÃO TRANSVERSAL

TABELA 28 - RESULTADOS DOS TESTES DE FLEXÃO ESTÁTICA EM RELAÇÃO AO EIXO DE MAIOR INÉRCIA PARA A CLASSE N²-ND.................................................. 82

TABELA 29 - RESULTADOS DOS TESTES DE FLEXÃO ESTÁTICA EM RELAÇÃO AO EIXO DE MAIOR INÉRCIA PARA A CLASSE N²-D. .................................................... 82

TABELA 30 - RESULTADOS DOS TESTES DE FLEXÃO ESTÁTICA EM RELAÇÃO AO EIXO DE MAIOR INÉRCIA PARA A CLASSE SS - ND. 
TABELA 31 - RESULTADOS DOS TESTES DE FLEXÃO ESTÁTICA EM RELAÇÃO AO EIXO DE MAIOR INÉRCIA PARA A CLASSE SS-D.

TABELA 32 - ESTATÍSTICA DESCRITIVA DOS RESULTADOS DO MOR OBTIDOS NOS TESTES DE FLEXÃO ESTÁTICA EM RELAÇÃO AO EIXO DE MAIOR INÉRCIA..............84

TABELA 33 - ESTATÍSTICA DESCRITIVA DOS RESULTADOS DO MOE OBTIDOS NOS TESTES DE FLEXÃO ESTÁTICA EM RELAÇÃO AO EIXO DE MAIOR INÉRCIA..............84

TABELA 34 - RESULTADOS DOS TESTES DE COMPRESSÃO PARALELA PARA A CLASSE $\mathrm{N}^{\mathrm{o}} 2$ - ND.

TABELA 35 - RESULTADOS DOS TESTES DE COMPRESSÃO PARALELA PARA A CLASSE No2 - D.

TABELA 36 - RESULTADOS DOS TESTES DE COMPRESSÃO PARALELA PARA A CLASSE SS - ND

TABELA 37 - RESULTADOS DOS TESTES DE COMPRESSÃO PARALELA PARA A CLASSE SS - D.

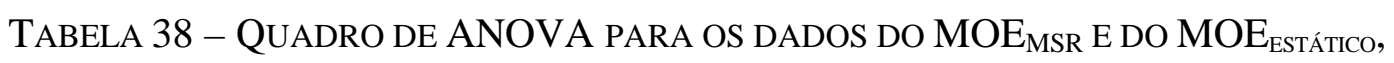
TRANSFORMADOS.

TABELA 39 - QUADRO DE ANOVA PARA OS DADOS DE $E_{M}$ E $F_{M}$ TRANSFORMADOS ...98

TABELA 40 - VALORES DO QUADRO DE ANOVA PARA OS DADOS DO MOE DA CLASSE $\mathrm{N}^{\mathrm{o}} 2$-ND.

TABELA 41 - TESTE T PARA OS DADOS PAREADOS DO MOE PARA A CLASSE N²-ND.

TABELA 42 - VALORES DO QUADRO DE ANOVA PARA OS DADOS TRANSFORMADOS DO MOE DA CLASSE N²-D.

TABELA 43 - TESTE T PARA OS DADOS PAREADOS DO MOE PARA A CLASSE N²-D. 104

TABELA 44 - VALORES DO QUADRO DE ANOVA PARA OS DADOS TRANSFORMADOS DO MOE DA CLASSE SS-ND.

TABELA 45 - TESTE T PARA OS DADOS PAREADOS DO MOE PARA A CLASSE SS-ND.

TABELA 46 - VALORES DO QUADRO DE ANOVA PARA OS DADOS TRANSFORMADOS DO MOE DA CLASSE SS-D.

TABELA 47 - TESTE T PARA OS DADOS PAREADOS DO MOE PARA A CLASSE SS-D ... 106

TABELA 48 - VALORES DO QUADRO DE ANOVA PARA OS DADOS DO MOR TRANSFORMADOS.

TABELA 49 - COMPARAÇÕES MÚLTIPLAS PAREADAS PARA AS MÉDIAS DO MOE TRANSFORMADO.

TABELA 50 - VALORES DO QUADRO DE ANOVA PARA OS DADOS DO MOR TRANFORMADOS.

TABELA 51 - COMPARAÇÕES MÚLTIPLAS PAREADAS PARA AS MÉDIAS DO MOR TRANSFORMADO.

TABELA 52 - QUADRO DE ANOVA PARA OS DADOS DE $E_{M}$ E $F_{M}$ TRANSFORMADOS .118

TABELA 53 - QUADRO DE ANOVA PARA OS DADOS DO MOR E DO MOE EM RELAÇÃO AO EIXO DE MENOR INÉRCIA.

TABELA 54 - TRANSFORMAÇÕES PARA OS DADOS DO MOE EM RELAÇÃO AO EIXO DE MAIOR INÉRCIA.

TABELA 55 - PROPRIEDADES DE RESISTÊNCIA E RIGIDEZ À FLEXÃO PARA A AMOSTRA

EM ESTUDO E PARA O SOUTHERN PINE NORTE-AMERICANO.

TABELA 56 - QUADRO DE ANOVA PARA OS DADOS DE E $\mathrm{CO}_{\mathrm{CO}}$ E $F_{\mathrm{CO}}$. 
TABELA 57 - QUADRO DE ANOVA PARA OS DADOS DE MOE E $F_{\mathrm{CO}}$ TRANSFORMADOS

TABELA 58 - QUADRO DE ANOVA PARA OS DADOS DE E $\mathrm{E}_{\mathrm{CO}} \mathrm{E} \rho_{\mathrm{AP}} \ldots \ldots \ldots \ldots \ldots \ldots \ldots \ldots . . . . . . . . . . . . .130$

TABELA 59 - RESULTADOS DOS TESTES DE COMPRESSÃO PARALELA......................... 132

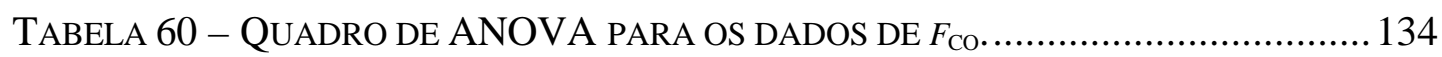

TABELA 61 - COMPARAÇÕES MÚLTIPLAS PAREADAS PARA AS MÉDIAS DO MOE

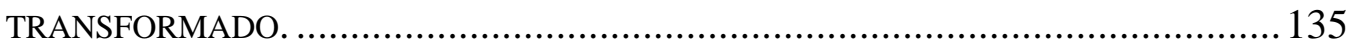

TABELA 62 - LIMITAÇÃO DA DIMENSÃO DOS NÓS EM FUNÇÃO DA PROPORÇÃO DA ÁREA DA SEÇÃO TRANSVERSAL OCUPADA. ....................................................... 137

TABELA 63 - PROPRIEDADES MECÂNICAS DE PEÇAS ESTRUTURAIS DE PINUS TAEDA E ELLIOTTII AGRUPADOS, E COM DIMENSÕES NOMINAIS DE 3,5CM X 12,5 CM. ...... 138

TABELA 64 - EQUAÇÕES DE CORRELAÇÃO ENTRE PROPRIEDADES MECÂNICAS E FÍSICAS PARA A AMOSTRA COLETADA. ................................................................... 140

TABELA 65- RAZÃO DE RESISTÊNCIA ASSOCIADA COM A INCLINAÇÃO DAS FIBRAS... 142

TABELA 66 - MÁXIMA INCLINAÇÃO DAS FIBRAS EM CADA CLASSE. .......................... 142

TABELA 67 - MÁXIMA PROPORÇÃO PERMISSÍVEL PARA OS NÓS NA FACE ESTREITA.. 143

TABELA 68 - LIMITAÇÕES PARA RACHAS E FENDAS.............................................. 145

TABELA 69 - ENCURVAMENTO PERMISSÍVEL PARA PEÇAS COM ESPESSURA DE 38MM.

TABELA 70 - ENCANOAMENTO MÁXIMO ADMITIDO EM CADA CLASSE.

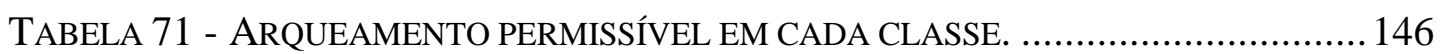

TABELA 72 - TORCIMENTO PERMISSÍVEL EM CADA CLASSE …............................... 147

TABELA 73 - TIPO DE RUPTURA. .................................................................. 149

TABELA 74 - EXEMPLO DE REGRAS DE CLASSIFICAÇÃO VISUAL PARA MADEIRA DE

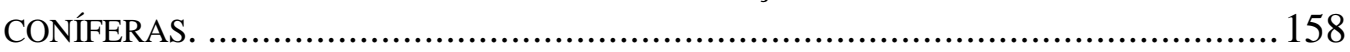

TABELA 75 - FATORES DE AJUSTE PARA RESISTÊNCIA DE CORPOS-DE-PROVA ISENTOS

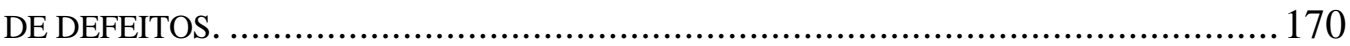

TABELA 76 - ÍNDICES DE RESISTÊNCIA À FLEXÃO EM TESTES COM GRANDES AMOSTRAS E VALORES DE MÓDULO DE ELASTICIDADE PARA MADEIRA CLASSIFICADA VISUALMENTE.

TABELA 77 - ÍNDICES DE RESISTÊNCIA À FLEXÃO E COMPRESSÃO PARALELA PARA MADEIRA CLASSIFICADA PELO SISTEMA E-RATED......................................... 171

TABELA 78 - FATORES MODIFICADORES DE TENSÃO ASSOCIADOS COM A INCLINAÇÃO DAS FIBRAS.

TABELA 79 - CONSTANTES USADAS PARA AJUSTAR A RELAÇÃO DE RESISTÊNCIA EM ELEMENTOS LAMINADOS VERTICALMENTE.................................................. 174

TABELA 80 - FATORES DE AJUSTE PARA O MÓDULO DE ELASTICIDADE...................... 174

TABELA 81 - FATORES MODIFICADORES MÍNIMOS PARA A RESISTÊNCIA À FLEXÃO E À COMPRESSÃO PARALELA ÀS FIBRAS PARA ELEMENTOS ESTRUTURAIS DE MLC PRODUZIDOS COM MADEIRA CLASSIFICADA PELO SISTEMA E-RATED................ 176

TABELA 82 - VALORES DE PROJETO PARA SOUTHERN PINE NORTE-AMERICANO....... 177 


\section{LISTA DE SIMBOLOS}

\section{Letras romanas maiúsculas}

AITC - American Institute of Timber Construction

ALSC - American Lumber Standard Committee

ANOVA - análise de variância

$\mathrm{C}_{\mathrm{D}}$ - coeficiente de duração do carregamento

$\mathrm{C}_{\mathrm{fu}}$ - coeficiente de posição

$\mathrm{C}_{\mathrm{F}}$ - coeficiente de dimensão

$\mathrm{C}_{\mathrm{M}}$ - coeficiente de umidade

$\mathrm{COV}$ - coeficiente de variação

$\mathrm{C}_{\mathrm{t}}$ - coeficiente de temperatura

${ }^{\circ} \mathrm{C}$ - temperatura em graus Celsius

E - módulo de elasticidade

EESC - Escola de Engenharia de São Carlos

$\mathrm{E}_{\mathrm{M} 12 \%}$ - módulo de elasticidade a $12 \%$ de umidade

$\mathrm{F}$ - força

FPL - Forest Products Laboratory

$\mathrm{F}_{\alpha ;(\mathrm{k}-1) ;(\mathrm{n}-\mathrm{k})}$ - valor tabelado para o teste $\mathrm{F}$

${ }^{\circ} \mathrm{F}$ - temperatura em graus Fahrenheit

$\mathrm{G}$ - módulo de elasticidade transversal

GPa -Giga Pascal

I - momento de inércia

$\mathrm{I}_{\mathrm{a}}$ - momento de inércia adotado

$\mathrm{I}_{\mathrm{r}}$ - momento de inércia real

$\mathrm{K}_{\text {mod3 }}$ - coeficiente parcial de modificação da norma NBR 7190-97

$\mathrm{L}$ - vão livre

LaMEM - Laboratório de Madeira e de Estruturas de Madeira

LVDT - Linear Variable Diferential Transformer

$\mathrm{L} / \mathrm{h}$ - razão entre o vão e a altura

MEL - Machine Evaluated Lumber

MOE - módulo de elasticidade

MOR - módulo de ruptura à flexão 
MPa -Mega Pascal

MSR - Machine Stress Rated

NDS - National Design Specification

NGR - National Grading Rule

NGRC - National Grading Rule Committee

$\mathrm{R}^{2}$ - coeficiente de determinação

Sd - desvio padrão

SET - departamento de estruturas

SPIB - Southern Pine Inspection Bureau

SR - razão de resistência

SQ - soma dos quadrados

USDA - United States Department of Agriculture

USP - Universidade de São Paulo

VQL - visual quality level

$\mathrm{W}-$ peso

\section{Letras romanas minúsculas}

b - base, a menor dimensão da seção transversal

$\mathrm{cm}$ - centímetro

$f$ - deslocamento vertical

$f_{\mathrm{c}}$ - resistência à compressão

$f_{\text {co }}$ - resistência a compressão paralela

$f_{d}$ - resistência de cálculo

$f_{\mathrm{k}}$ - resistência característica

$f_{\mathrm{m}}-$ módulo de ruptura à flexão

$f_{\mathrm{r}}$ - freqüência natural de vibração

$\mathrm{g}$ - aceleração da gravidade; ou unidade de massa em gramas

gl - grau de liberdade

$\mathrm{h}$ - altura, a maior dimensão da seção transversal

in - unidade de comprimento em polegadas (inches)

$\mathrm{k}$ - constante

$\mathrm{lb}$ - unidade de força em libra

$\ln$ - lognormal 
$\mathrm{m}-$ metro

$\min -$ minutos

mm - milímetro

$t_{\alpha / 2}-$ valor tabelado da distribuição $t$ de student para o nível de significância $\alpha / 2$

$\bar{x}$ - média das observações

$\mathrm{x}_{\mathrm{p}}-$ resposta prevista

$\mathrm{x}_{\mathrm{ti}}-$ valor observado

$\mathrm{x}_{\mathrm{ti}}{ }^{\prime}$ - valor estimado

\section{Letras gregas minúsculas}

$\alpha$ - nível de significância; ou termo independente

$\beta$ - coeficiente angular

$\varepsilon$ - deformação específica

$\varepsilon_{\mathrm{i}}$ - resíduo para a observaçãoi

$\lambda$ - coeficiente para a transformação de Box-Cox

$\mu$ - média

$\rho_{\mathrm{ap}}-$ densidade aparente

\section{Letras gregas minúsculas}

$\Delta$ - deslocamento vertical

$\Delta P$ - incremento de força

$\Delta v$ - incremento de deslocamento vertical

$\sigma$ - tensão normal

$\sigma^{2}-$ variância 


\section{RESUMO}

CARREIRA, M. R. (2003). Critérios para classificação visual de peças estruturais de Pinus Sp. São Carlos. 182p. Dissertação (Mestrado) - Escola de engenharia de São Carlos, Universidade de São Paulo.

A utilização estrutural da madeira serrada oriunda de florestas plantadas apresenta, no Brasil, um considerável potencial para expansão, pois as restrições sobre as florestas nativas são irreversíveis e o mercado se volta, em larga escala, para o uso das madeiras de reflorestamento, destacando-se o Pinus $S p$ como uma das espécies mais promissoras. Entretanto, como resultado do crescimento acelerado, a madeira proveniente destas espécies apresenta muitos defeitos, tornando-se imprescindível a realização da classificação para sua aplicação estrutural. Por outro lado, atualmente no Brasil não há procedimentos normatizados para a classificação de peças estruturais de madeira. Neste trabalho foi verificada a adequabilidade das regras de classificação visual do Southern Pine Inspection Bureau (SPIB) aplicadas à madeira de Pinus provenientes de florestas plantadas na região de Lages, Santa Catarina. Foi realizada a classificação visual de 600 peças de Pinus Sp. que tiveram a sua rigidez à flexão em relação ao eixo de menor inércia determinada por três métodos: ensaio estático de flexão, equipamento MSR, e equipamento de vibração transversal. Parte destas peças (96) distribuídas em igual número em quatro classes de resistência foi testada à flexão estática em relação ao eixo de maior inércia, com a finalidade de determinar a resistência e a rigidez à flexão de cada classe. O trabalho apresenta correlações entre propriedades de resistência, rigidez e a densidade. $\mathrm{O}$ método de classificação visual descrito nas regras do SPIB mostrou-se adequado para ser aplicado na madeira proveniente de florestas de Pinus $S p$ plantadas no Brasil.

Palavras-chave: madeiras, classificação visual, Pinus. 


\begin{abstract}
CARREIRA, M. R. (2003). Rules for visual grading of structural lumber of Pinus Sp. São Carlos. 182p. Dissertação (Mestrado) - Escola de engenharia de São Carlos, Universidade de São Paulo.
\end{abstract}

The structural use of lumber from planted forests presents, in Brazil, a considerable potential for expansion, whereas the restrictions on the native forests are irreversible and the market turns, in wide scale, for the use of the reforestation lumber, standing out the lumber of Pinus $S p$ as one of the most promising species. However, as a result of the fast growth, the coming lumber of these species presents many defects, becoming indispensable the accomplishment of the grading for its structural application. On the other hand, now in Brazil there are not established procedures for the grading of structural lumber. In this work the adequability of Southern Pine Inspection Bureau (SPIB) grading rules applied to lumber of reforestation from city of Lages - SC, were verified. The visual grading of 600 pieces of Pinus $S p$ was accomplished. The flatwise bending stiffness was determined by three methods: static bending, MSR equipment, and equipment of transverse vibration. A part of these pieces (96) distributed in the same number in four structural grades it was tested to the static edgewise bending, with the purpose of determining bending strength and stiffness of each grade. This work presents correlations among strength properties, stiffness and the density. The method of visual grading described in the rules of SPIB was shown appropriate to be applied in the lumber from forests of Pinus Sp planted in Brazil.

Keywords: lumber, visual grading, Pinus. 


\section{INTRODUÇÃO}

A madeira serrada de uma tora, independentemente da espécie e dimensão, é bastante variável em suas propriedades mecânicas. É comum encontrar diferenças de resistência acima de trezentos por cento de uma peça para outra da mesma espécie e mesma dimensão. Segundo CURRY (1969), há duas razões para isto. A primeira razão se deve à forte correlação existente entre a resistência e a densidade da madeira. Há uma grande variação nesta característica entre as árvores de uma mesma espécie. Ainda de acordo com CURRY (1969), a segunda razão é que outras características naturais de crescimento, em particular os nós, têm um efeito adverso na resistência, e a extensão, localização e a dimensão deles diferem em cada peça de madeira.

Por outro lado, o máximo de economia do material é obtido quando a variação das características em uma determinada classe é limitada, e todas as peças são utilizadas com seu total potencial.

A norma brasileira NBR 7190-97 (Projeto de Estruturas de Madeira) não apresenta os procedimentos para a classificação estrutural de peças de madeira. Porém, a qualidade da madeira é levada em conta no dimensionamento por meio do coeficiente parcial de modificação $K_{\text {mod3 }}$. Este coeficiente é adotado igual a 1,0 no caso de madeira de primeira categoria, e igual a 0,8 no caso de madeira de segunda categoria. A condição de madeira de primeira categoria descrita na norma NBR 7190-97 somente pode ser admitida se todas as peças estruturais forem classificadas como isentas de defeitos, por meio de método visual normatizado, e também submetidas a uma classificação mecânica que garanta a homogeneidade da rigidez das peças que compõem o lote. Desta forma, torna-se necessário agrupar as peças de madeira com propriedades semelhantes em classes, permitindo assim, a utilização segura e racional dos recursos florestais disponíveis. 
Neste escopo, foi aplicado neste trabalho o método norte-americano de classificação visual descrito nas regras de classificação visual do Southern Pine Inspection Bureau (SPIB) com base na norma ASTM D245-93 (Standard Practice for Establishing Structural Grades and Related Allowable Properties for Visually Graded Lumber) para a classificação estrutural de peças de madeira do gênero Pinus e verificação da adequação deste método às espécies de Pinus Sp plantadas no Brasil. Determinou-se também o valor característico do módulo de ruptura à flexão para cada classe visual por meio de análise estatística da distribuição de resistência de cada classe.

\subsection{Objetivo}

Os objetivos principais deste trabalho compreendem a verificação da eficiência e da adequação das regras de classificação visual do SPIB aplicadas à madeira de coníferas das espécies Pinus elliottii e Pinus taeda provenientes de florestas plantadas no Brasil e determinar valores de propriedades de resistência e de rigidez para o lote em estudo.

Os objetivos secundários são:

- Verificar a influência da disposição das peças na determinação da rigidez à flexão;

- Aferir os resultados da máquina MSR e do equipamento de vibração transversal;

- Correlacionar o módulo de ruptura em relação ao eixo de maior inércia com a rigidez à flexão em relação aos eixos de maior e de menor inércia;

- Correlacionar a resistência à compressão paralela em corpos-deprova com a rigidez à compressão paralela;

- Correlacionar a resistência à compressão paralela em corpos-deprova com a densidade

- Correlacionar a rigidez à compressão paralela em corpos de prova com a densidade. 